\title{
Editorial
}

\section{Laparoscopic-Assisted Proctectomy for Rectal Cancer: On Trial}

\author{
Mattias Soop, MD, PhD and Heidi Nelson, MD \\ Department of Surgery, Division of Colon and Rectal Surgery, Mayo Clinic, 200 First Street SW, Rochester,
} MN 55901, USA

\section{THE QUESTION}

In 2005, the American Society of Colon and Rectal Surgeons (ASCRS) issued a statement regarding the role of laparoscopy in proctectomy for cancer: "The absence of five-year survival data makes it premature to endorse laparoscopic proctectomy for curable cancer." ${ }^{1}$ In this issue, $\mathrm{Ng}$ and colleagues ${ }^{2}$ provide new evidence in support of the short-term advantages for laparoscopic proctectomy and the long-term equivalence in oncologic outcomes. The question of the day for laparoscopic colorectal surgery is, therefore, whether the current study by $\mathrm{Ng}$ et al. and other recent literature provide the strength of evidence necessary to revise the 2005 ASCRS practice parameters on laparoscopic proctectomy for cancer. As we will discuss below, we believe that the cumulative literature now supports a state of equipoise on short-term advantages and long-term oncologic outcomes, making the case for large prospective randomized trials.

\section{CURRENT LITERATURE}

Since 2005, new literature has become available from at least four key sources including the latest Annals of Surgical Oncology report from $\mathrm{Ng}$ et al. Subgroup analyses of rectal cancer patients are reported from two randomized trials: the Laparoscopic-Assisted Surgery In Colorectal Cancer (CLA-

Published online July 10, 2008.

Address correspondence and reprint requests to: Heidi Nelson, MD; E-mail: nelson.heidi@mayo.edu

Published by Springer Science+Business Media, LLC (C) 2008 The Society of Surgical Oncology, Inc.
SICC) trial $(n=381)^{3}$ and a single-center trial from Dr Ng's group at the Chinese University of Hong Kong $(n=153))^{4,5}$ In addition, a meta-analysis of mainly nonrandomized studies $(n=2071)$ has been reported. ${ }^{6}$ In the present study by $\mathrm{Ng}$ and colleagues, 99 patients with rectal cancer within $5 \mathrm{~cm}$ from the anal verge were randomized to undergo abdominal perineal resection by either an open approach or by a truly laparoscopic approach, i.e., with pelvic dissection performed under pneumoperitoneum. Their conversion rate $(9.8 \%)$ is a third of the conversion rates in their prior trial $(30.3 \%)^{4}$ and in the CLASICC trial $(34 \%),{ }^{3}$ indicating that, while laparoscopic pelvic surgery is technically demanding, results will improve as experience accumulates. Although the numbers of patients studied are small, these three trials and the meta-analysis offer important and timely estimates of both the short-term advantages and long-term oncologic outcomes for laparoscopic proctectomy.

\section{SHORT-TERM ADVANTAGES}

The most consistent advantage for laparoscopic proctectomy in the literature, including the current paper by $\mathrm{Ng}$ et al., is an improvement in physiological recovery, not dissimilar to what was seen for laparoscopic colectomy. ${ }^{7}$ Thus, time to first bowel or stoma movement has typically been found to be reduced by $1-2$ days, ${ }^{2,3,6}$ time to oral diet by 1 day, ${ }^{2,5,6}$ and time to independent mobilization by $1-1.5$ days. ${ }^{2,5}$ This improved physiologic recovery, however, has not translated into consistent reductions in hospital stay, which remain long (around 10 days) regardless of surgical approach in the 
randomized trials, including the present paper by $\mathrm{Ng}$ and colleagues, ${ }^{2,3,5}$ underscoring the often observed discrepancy between time to physiologic recovery after surgery and actual discharge from hospital. A significant reduction of hospital stay (2.7 days) was demonstrated in the meta-analysis, ${ }^{6}$ suggesting that this is achievable.

Perioperative care and tradition are important determinants of short-term outcomes. In the trials so far, perioperative care has been conservative. It is possible that a comprehensive perioperative enhancedrecovery program, consisting of elements known to blunt the physiological stress response to surgery, support postoperative organ function, and decrease morbidity, ${ }^{8}$ would bring to light more significant shortterm advantages from minimally invasive surgery.

\section{ONCOLOGIC OUTCOMES}

The first report from the CLASICC trial raised serious concerns regarding the adequacy of laparoscopic proctectomy, reporting higher rates of positive circumferential resection margins for laparoscopic anterior resection $(16 / 129$ or $12 \%)$ as compared with open anterior resection $(4 / 64$ or $6 \%){ }^{3}$ Guillou and colleagues advised against the routine practice of laparoscopic anterior resection based on these findings. In the present study by $\mathrm{Ng}$ et al., the rates of circumferential resection margin involvement for low rectal cancer cases were similar in the laparoscopic $(3 / 51$ or $6 \%)$ and in the open $(2 / 48$ or $4 \%)$ groups. The meta-analysis of controlled studies also did not show a difference in positive margins. ${ }^{6}$ It is not clear yet whether the concern for positive circumferential margins has been definitively resolved. In part, the trials we mention attempt to address this concern by providing long-term oncologic results.

The median period of follow-up in the present study by $\mathrm{Ng}$ and colleagues was over 7 years, with only two patients lost to follow-up. In this relatively small study, there were no differences between groups in local recurrence rates $(5 \%$ versus $11.1 \%$ in the laparoscopic versus the open group, respectively), similar in magnitude to 3-year data in rectal cancer patients from the CLASICC trial $(9.7 \%$ versus $10.1 \%) .{ }^{9}$ Overall survival was also no different between groups in the present study at 5 years $(75.2 \%$ versus $76.5 \%)$ or in the CLASICC trial at 3 years $(65.2 \%$ versus $57.7 \%)$. In total, roughly 630 patients contribute prospective long-term data on cancer outcomes following laparoscopic versus open surgery for rectal cancer. $\mathrm{Ng}$ and colleagues acknowledge that a randomized clinical trial of approximately 4,000 patients would be needed to demonstrate that long-term survival is no different between laparoscopic and open proctectomy. ${ }^{2}$

Taken together, the data from the present and the two previously published randomized clinical trials have taken laparoscopic proctectomy to a state of equipoise, where significant short-term benefits are likely, and significant oncologic compromise is less likely. However, an advantageous risk-benefit ratio remains to be proven. Adequately powered, multicenter trials are therefore justified and necessary at this point to reach a conclusion about the role of laparoscopy in rectal cancer surgery. For this reason, laparoscopic proctectomy for cancer should remain a procedure under evaluation, and should only be performed within the context of prospectively evaluated clinical trials.

\section{CURRENT TRIALS}

Around the world, three large multicenter trials are presently or imminently open: the European COLOR II and the Japanese JCOG 0404 trials, as well as the upcoming National Cancer Institutes-sponsored American College of Surgeons Oncology Group (ACOSOG) Z6051 trial that is set to open in the USA this year.

The ACOSOG Z6051 trial is a multicenter, phase III, randomized clinical trial of laparoscopic-assisted resection for T1-3N1M0 or T3N0M0 rectal cancers with the lower edge $\leq 12 \mathrm{~cm}$ from the anus by rigid proctoscopy. This noninferiority trial has as its primary endpoint a composite endpoint based on detailed and standardized pathologic evaluation of the specimen, including circumferential and distal margins and the completeness of the total mesorectal excision. This primary endpoint is a novel, surrogate endpoint for long-term oncologic outcome; it will reduce both the necessary accrual target of the trial and its time to maturation. Secondary endpoints include 2-year local recurrence rates and quality-of-life measures. Surgeon credentialing will be based on having completed 20 laparoscopic-assisted resections each of colon and rectal cancers, including one video assessment of pelvic dissection. Surgical techniques will be standardized and require that the pelvic dissection be performed under pneumoperitoneum. Surgeons who are interested in joining the Z6051 trial are invited to contact the ACOSOG Membership Coordinator at harbe001@notes.duke.edu or the corresponding author for more details. 


\section{CONCLUSIONS}

The present study by $\mathrm{Ng}$ et al. thus levels the balance between possible benefits and risks of laparoscopic proctectomy. This report increases the likelihood that future trials will confirm the clinical benefits and the oncologic equivalence of laparoscopic proctectomy. Forthcoming phase III trials will more definitively evaluate whether laparoscopic proctectomy will emerge as standard of care in the future and perhaps revise the practice parameters. Until then, we encourage surgeons to sign up to participate in the ACOSOG Z6051 trial or similar local, national, or international trials.

\section{REFERENCES}

1. Tjandra JJ, Kilkenny JW, Buie WD, et al. Practice parameters for the management of rectal cancer (revised). Dis Colon Rectum 2005; 48:411-23.
2. Ng SS, Leung KL, Lee JF, et al. Laparoscopic-assisted versus open abdominoperineal resection for low rectal cancer: a prospective randomized trial. Ann Surg Oncol 2008; [Epub ahead of print].

3. Guillou PJ, Quirke P, Thorpe H, et al. Short-term endpoints of conventional versus laparoscopic-assisted surgery in patients with colorectal cancer (MRC CLASICC trial): multicentre, randomised controlled trial. Lancet 2005; 365:1718-26.

4. Leung KL, Kwok SP, Lam SC, et al. Laparoscopic resection of rectosigmoid carcinoma: prospective randomised trial. Lancet 2004; 363:1187-92.

5. Ng SS, Leung KL, Lee JF, et al. MRC CLASICC trial. Lancet $2005 ; 366: 713$

6. Aziz O, Constantinides V, Tekkis PP, et al. Laparoscopic versus open surgery for rectal cancer: a meta-analysis. Ann Surg Oncol 2006; 13:413-24.

7. Nelson H, Sargent DJ, Wieand S, et al. A comparison of laparoscopically assisted and open colectomy for colon cancer. $N$ Engl J Med 2004; 350:2050-9.

8. Kehlet H. Fast-track colorectal surgery. Lancet 2008; 371:791-3.

9. Jayne DG, Guillou PJ, Thorpe H, et al. Randomized trial of laparoscopic-assisted resection of colorectal carcinoma: 3-year results of the UK MRC CLASICC Trial Group. J Clin Oncol 2007; 25:3061-8. 